11 Schaub B, Westlake RM, He $\mathrm{H}$, et al. Surfactant protein D deficiency influences allergic immune responses. Clin Exp Allergy 2004; 34: 1819-1826.

12 Ledford JG, Addison KJ, Foster MW, et al. Eosinophil-associated lung diseases. A cry for surfactant proteins A and D help? Am J Respir Cell Mol Biol 2014; 51: 604-614.

13 Ofek I, Mesika A, Kalina M, et al. Surfactant protein D enhances phagocytosis and killing of unencapsulated phase variants of Klebsiella pneumoniae. Infect Immun 2001; 69: 24-33.

14 Scichilone N, Marchese R, Soresi S, et al. Deep inspiration-induced changes in lung volume decrease with severity of asthma. Respir Med 2007; 101: 951-956.

15 Pyrgos G, Scichilone N, Togias A, et al. Bronchodilation response to deep inspirations in asthma is dependent on airway distensibility and air trapping. J Appl Physiol 2011; 110: 472-479.

\title{
Incorporating therapeutic drug monitoring into the World Health Organization hierarchy of tuberculosis diagnostics
}

\author{
To the Editor:
}

Tuberculosis (TB), once considered as a disease of the past generally afflicting poor people, still claims 1.5 million lives annually [1]. Although $86 \%$ of patients with drug susceptible TB are cured with established first-line drugs, treatment is often longer than 6 months due to slow response, compliance problems or adverse drug reactions. In addition, emergence of drug-resistant Mycobacterium tuberculosis strains with an unacceptably low treatment success rate of $50 \%$ and TB-HIV co-infection have challenged the goals of global TB control and elimination [1].

Pharmacokinetic variability is a major driver of acquired drug resistance due to comorbidities, co-medications and intra-individual differences [2]. Therefore, monitoring the exposure-response relationship by incorporating pharmacokinetics and minimum inhibitory concentration (MIC) of the anti-TB drugs would conceivably help combat the current challenges of drug resistance, toxicity, relapse and nonresponse [3]. Drug exposure over time (area under the concentration-time curve $\left(\mathrm{AUC}_{0-24}\right)$ ) and peak serum concentration $\left(\mathrm{C}_{\max }\right)$ are the two parameters that, in combination with MIC, predict development of acquired drug resistance and are expressed as a ratio of $\mathrm{AUC}_{0-24} / \mathrm{MIC}$ or $\mathrm{C}_{\max } / \mathrm{MIC}$ [4]. For instance, a patient with serum concentrations below the suggested therapeutic threshold may still achieve a successful treatment outcome because of a low MIC of the offending organism [5]. However, patients with altered pharmacokinetic parameters (e.g. because of comorbidities), abnormally low body mass index, low $\mathrm{AUC}_{0-24}$ and high MIC values are at the peril of treatment failure [6].

For the measurement of drug concentrations in areas with limited resources, dried blood spot (DBS) sampling can be introduced as an easy sampling procedure. In DBS sampling, whole blood is obtained via finger prick and dropped onto a sampling paper which is dried, extracted and then analysed by validated methods such as liquid chromatography-tandem mass spectrometry (LC-MS/MS). DBS sampling overcomes the costs and logistic problems related to venous blood sampling such as larger sample volume, invasion with needles, storage conditions, transportation and biohazard risks. Finally, this technology is highly appropriate for children affected with TB. Obtaining a full time concentration profile for $\mathrm{AUC}_{0-24} /$ MIC ratio is not feasible in the rural clinics. Therefore, limited sampling strategies could be applied to estimate the total exposure [7].

To optimise drug therapy, therapeutic drug monitoring (TDM) has become a standard clinical technique, as agreed among researchers and healthcare policy makers [8]. Despite broad acceptance, TDM has not yet been implemented in resource constrained countries with a high TB burden [9]. This might be due to the limited budget the health sector receives and the lack of advanced bioanalytical infrastructure for performing TDM. To overcome this problem we propose to organise the logistics for TDM in a similar way as for TB diagnostics. Here we describe the tools and strategy for implementing TDM at three levels (figure 1). 


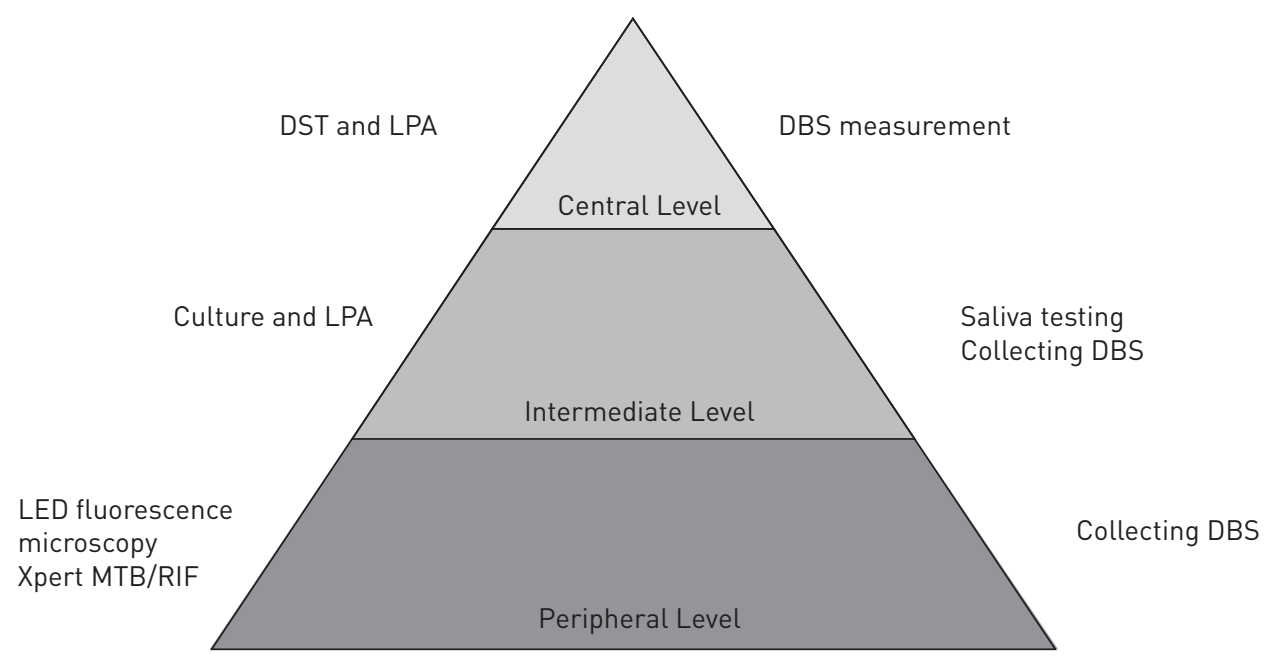

FIGURE 1 Implementation of therapeutic drug monitoring (TDM) into the three tiers of the World Health Organization pyramid of tuberculosis diagnostics. Please refer [16] for detailed information about diagnostic techniques in a tiered laboratory network. DBS: dried blood spot; LED: light-emitting diode; LPA: line probe assay; DST: drug susceptibility testing.

First, at a peripheral (community) level, DBS sampling can be used to collect the blood samples. DBS samples will be collected in the second week of treatment and both healthcare workers and patients can perform the finger prick themselves, after appropriate training and with easy-to-follow instructions. Afterwards the sample can be dispatched to the laboratory at a central level, where the analysis takes place using LC-MS/MS.

To implement the proposed TDM intervention at this level funds are needed for the salaries of healthcare personnel and DBS kits, and for training, continued education and supervision. Furthermore, for simultaneous analysis of multiple anti-TB drugs in a DBS sample, a new LC-MS/MS method can be developed and validated similar to the one published by KIM et al. [10] for human plasma using an LC-MS/MS method [11].

Secondly, the intermediate level consists of a decentralised laboratory service, which can use semi-quantitative thin layer chromatography (TLC) methods to detect the concentration of cornerstone drugs like rifampicin and fluoroquinolones in saliva of TB patients $[12,13]$. This simple, affordable and noninvasive point-of-care test will be able to detect patients with low drug exposure to key anti-TB drugs fast, while waiting for the results of DBS samples that are also collected at this level. The benefit of this level is the ability for TDM-guided dosing for patients with low drug exposure.

Thus, availability of both TLC and DBS results will make laboratories self-sufficient to perform TDM, in contrast to the laboratories at the peripheral level that will solely depend on the central level laboratory for DBS sample results. It is also possible that the costs (medical and laboratory) will be relatively higher when compared with the peripheral level. For instance, additional laboratory costs would include purchasing TLC plates, solvents and ultraviolet lights, and costs for training and salaries. The return of this investment may be high if treatment is adjusted in a timely manner for patients with a complicated course, severe disease and disease progression during treatment.

Thirdly and finally, to support a clinical TDM service, the central level laboratory will analyse DBS samples collected from intermediate and peripheral laboratories using advanced techniques such as LC-MS/MS. The laboratories at the central level in most of the low- and middle-income countries are generally well-equipped, and would be able to handle a high sample throughput demanding limited extra resources. Extra resources might comprise instalment of an LC-MS/MS and better staffing to efficiently handle an increased sample load. Test results from central to intermediate and peripheral laboratories can be communicated utilising the existing framework. Furthermore, supranational TB reference laboratories can support the strengthening of central/national reference laboratories by establishing a working relationship, providing technical assistance, assisting in the setup and validation of infrastructure and equipment, and facilitating human resources development. Quality control procedures will increase laboratory credibility and also boost the confidence of clinicians, researchers, accrediting bodies and regulatory agencies [14]. 
TDM, although potentially highly valuable, is one of the least acknowledged tools in international TB treatment guidelines. Currently, TDM is merely recommended as an option mainly based on perceived cost constraints, lack of infrastructure and trained personnel [15]. However, ineffective or incomplete treatment, slow drug responses leading to prolonged infectiousness, acquired drug resistance, treatment failure and early relapse, as well as the emergence of multidrug-resistant TB that all thrive in the absence of TDM, call for a change to bring TDM to the forefront [15]. TDM could be cost-effective even in high incidence, low resource settings [3]. With the novel tools and procedures in place, TDM should no longer be a remote possibility but rather be adapted as an integral component of national TB programmes similar as TB diagnostics and first- and second-line TB drug supply. http://ow.ly/YcRMw

Samiksha Ghimire ${ }^{1}$, Mathieu S. Bolhuis ${ }^{1}$, Marieke G.G. Sturkenboom ${ }^{1}$, Onno W. Akkerman ${ }^{2,3}$, Wiel C.M. de Lange ${ }^{2,3}$, Tjip S. van der Werf ${ }^{2,4}$ and Jan-Willem C. Alffenaar ${ }^{1}$

${ }^{1}$ University of Groningen, University Medical Center Groningen, Dept of Clinical Pharmacy and Pharmacology, Groningen, The Netherlands. ${ }^{2}$ University of Groningen, University Medical Center Groningen, Dept of Pulmonary Diseases and Tuberculosis, Groningen, The Netherlands. ${ }^{3}$ University of Groningen, University Medical Center Groningen, Tuberculosis Center Beatrixoord, Haren, The Netherlands. ${ }^{4}$ University of Groningen, University Medical Center Groningen, Dept of Internal Medicine, Groningen, The Netherlands.

Correspondence: Jan-Willem C. Alffenaar, University Medical Center Groningen, Dept of Clinical Pharmacy and Pharmacology, PO box 30.001, 9700 RB Groningen, The Netherlands. E-mail: j.w.c.alffenaar@umcg.nl

Received: Nov 062015 | Accepted after revision: Jan 282016 | First published online: March 172016

Conflict of interest: None declared.

\section{References}

1 World Health Organization (WHO). Global Tuberculosis Report 2015 (20th Edition). Geneva, WHO Press, 2015.

2 Srivastava S, Pasipanodya JG, Meek C, et al. Multidrug-resistant tuberculosis not due to noncompliance but to between-patient pharmacokinetic variability. J Infect Dis 2011; 204: 1951-1959.

3 van der Burgt EP, Sturkenboom MG, Bolhuis MS, et al. End TB with precision treatment! Eur Respir J 2016; 47: 680-682.

4 Gumbo T, Pasipanodya JG, Wash P, et al. Redefining multidrug-resistant tuberculosis based on clinical response to combination therapy. Antimicrob Agents Chemother 2014; 58: 6111-6115.

5 Daskapan A, de Lange WC, Akkerman OW, et al. The role of therapeutic drug monitoring in individualised drug dosage and exposure measurement in tuberculosis and HIV co-infection. Eur Respir J 2015; 45: 569-571.

6 Pranger $\mathrm{AD}$, van Altena $\mathrm{R}$, Aarnoutse $\mathrm{RE}$, et al. Evaluation of moxifloxacin for the treatment of tuberculosis: 3 years of experience. Eur Respir J 2011; 38: 888-894.

7 Alsultan A, An G, Peloquin CA. Limited sampling strategy and target attainment analysis for levofloxacin in patients with tuberculosis. Antimicrob Agents Chemother 2015; 59: 3800-3807.

8 Alsultan A, Peloquin CA. Therapeutic drug monitoring in the treatment of tuberculosis: an update. Drugs 2014; 74: 839-854.

9 Srivastava S, Peloquin CA, Sotgiu G, et al. Therapeutic drug management: is it the future of multidrug-resistant tuberculosis treatment? Eur Respir J 2013; 42: 1449-1453.

10 Kim HJ, Seo KA, Kim HM, et al. Simple and accurate quantitative analysis of 20 anti-tuberculosis drugs in human plasma using liquid chromatography-electrospray ionization-tandem mass spectrometry. J Pharm Biomed Anal 2015; 102: 9-16.

11 Hofman S, Bolhuis MS, Koster RA, et al. Role of therapeutic drug monitoring in pulmonary infections: use and potential for expanded use of dried blood spot samples. Bioanalysis 2015; 7: 481-495.

12 Orisakwe OE, Akunyili DN, Agbasi PU, et al. Some plasma and saliva pharmacokinetics parameters of rifampicin in the presence of pefloxacin. Am J Ther 2004; 11: 283-287.

13 Kozjek F, Šuturkova LJ, Antolič G, et al. Kinetics of 4-fluoroquinolones permeation into saliva. Biopharm Drug Dispos 1999; 20: 183-191.

14 Aarnoutse RE, Sturkenboom MG, Robijns K, et al. An interlaboratory quality control programme for the measurement of tuberculosis drugs. Eur Respir J 2015; 46: 268-271.

15 Heysell SK, Moore JL, Keller SJ, et al. Therapeutic drug monitoring for slow response to tuberculosis treatment in a state control program, Virginia, USA. Emerg Infect Dis 2010; 16: 1546-1553.

16 World Health Organization. Implementing tuberculosis diagnostics: policy framework. Geneva, WHO Press, 2015. 